\title{
Response to letter to the editor: "Remarks on Gobbi et al.: Two-year clinical outcomes of autologous micro-fragmented adipose tissue in elderly patients with knee osteoarthritis: a multi-centric, international study"
}

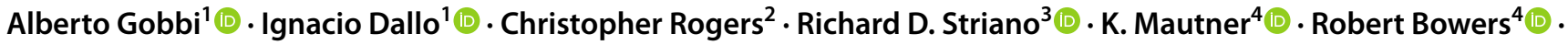 \\ Michael Rozak ${ }^{4}$ ( $)$ Norma Bilbool ${ }^{3} \cdot$ William D. Murrell ${ }^{5,6}$ (1)
}

Received: 4 May 2021 / Accepted: 7 May 2021 / Published online: 13 July 2021

(C) This is a U.S. government work and not under copyright protection in the U.S.; foreign copyright protection may apply 2021

\section{Dear Editor,}

We appreciate Dr. P. Wang et al.'s [1] comments regarding our article titled "Two-year clinical outcomes of autologous microfragmented adipose tissue in elderly patients with knee osteoarthritis: a multi-centric, international study" [2].

Their feedback on our multicentric international study has been truly appreciated. We continue to encourage other colleagues to provide their critical analysis on the continually growing interests in the use of adipose-derived cell-based therapies to treat osteoarthritis.

As stated by Wang et al., we agree that autologous microfragmented adipose tissue (MFAT) should not be considered the first-line treatment for KL 4 knee osteoarthritis (OA); however, many other factors must be considered, such as the patient's age, personal preferences, long waiting list, such as during the COVID-19 pandemic period, where patients could not be treated in the Hospitals, and also socio-economic issues; thus, other treatments should be considered.

We also wanted to demonstrate that patients with comorbidities might not be good candidates for arthroplasty; therefore, MFAT could represent a viable option.

\footnotetext{
William D. Murrell

doctormurrell@gmail.com

O.A.S.I Bioresearch Foundation Gobbi Onlus, Milan, Italy

2 San Diego Orthobiologics Medical Group, San Diego, CA, USA

3 Optimum Joint, Suffern, NY, USA

4 Emory Sports Medicine Center, Atlanta, GA, USA

5 Abu Dhabi Knee and Sports Medicine, Healthpoint Hospital, Zayed Sports City, Abu Dhabi, UAE

6 411th HC, Jacksonville, FL, USA
}

The purpose of our study was to evaluate the clinical outcomes of a single dose of MFAT injection in a retrospective multicentric study. Although we agree that the results would have provided additional insights regarding MFAT treatment, this study was not designed to evaluate MRI findings' changes. In particular, it has been shown that patients with bone marrow lesions on MRI have a significantly increased risk of total knee arthroplasty within three years [3]. We recommend that future studies will evaluate the correlation of baseline MRI studies with clinical outcomes; however, it is well known and often communicated, at least by the authors of this intervention, that treatment goals are not to regenerate cartilage but symptom amelioration through MSC-mediated immune-modulation and trophic activities. The MSC will dock at these sites, sense the local environment, and react by secreting agents based on cell types at that site. This in situ MSC secretes a curtain of bioactive agents that locally inhibit the over-aggressive immune system from sending in integrating cells. The MSC's "trophic" effects establish a regenerative microenvironment at the site of injury by:

A. Inhibiting ischemia-related apoptosis;

B. Inhibiting scar formation;

C. Stimulating angiogenesis by secreting large amounts of VEGF and by some of the MSC becoming pericytes again that function to stabilize the fragile, newly forming capillaries;

D. The MSC secreting tissue progenitor-specific mitogens so that the slow process of tissue regeneration is enhanced $[4,5]$.

Others have evaluated the relationship between metabolic factors such as diabetes, hypertension, and dyslipidemia 
with knee osteoarthritis [6]. We agree that future studies should evaluate medical conditions and medications which may impact the effects of treatments for knee osteoarthritis. Furthermore, the authors jointly agree that in future studies, characterization of injectates, cellular components, cellular counts, viability, and sterility are essential to identify product variabilities that could impact the outcome. Improved tracking of ortho-biologic intervention, especially safety and adverse events, and outcomes is being equally important.

We thank again Dr. Wang for the comments and look forward to furthering the investigation on adipose-derived cellbased therapies to treat osteoarthritis. Expressly, a longerterm, controlled, randomized study is warranted.

Supplementary Information The online version contains supplementary material available at https://doi.org/10.1007/s00264-021-05067-5.

Author contribution All authors analyzed and/or interpreted data. All authors collaborated in the drafting and critical revision of the manuscript. All authors approved the final version of the manuscript and vouch for the accuracy of the analysis and the fidelity of the study to the protocol.

\section{Funding Self-funded.}

Availability of data and material Underlying data from this manuscript may be requested by qualified researchers upon request. Investigators may request access to deidentified patient data and redacted study documents which may include raw datasets, analysis-ready data sets, and blank data forms. Prior to the use of data, proposals need to be approved by an independent review panel at www.clinicalstudyrequest. com and a signed data sharing agreement will need to be executed. Some documents are available in Italian, and others English.

CR-Medical Director and shareholder-Personalized Stem Cells, Inc, Co-Founder, and shareholder-DataBiologics, Inc.
Consent to participate All patients were approved for treatment by written informed consent.

Consent for publication Written informed consent was obtained from all patients.

Competing interests $\mathrm{CR}$, Consultant, Lipogems, Inc.

\section{References}

1. Wang P, Wang X, and Tong P (2021) Remark on Gobbi et al.: twoyear clinical outcomes of autologous microfragmented adipose tissue in elderly patients with knee osteoarthritis: a multi-centric, international study. Int Orthop. https://doi.org/10.1007/s00264021-05025-1. Advance online publication

2. Gobbi A, Dallo I, Rogers C, Striano RD, Mautner K, Bowers R, Rozak M, Bilbool N, and Murrell WD (2021) Two-year clinical outcomes of autologous microfragmented adipose tissue in elderly patients with knee osteoarthritis: a multi-centric, international study. Int Orthop. https://doi.org/10.1007/s00264-021-04947-0. Advance online publication

3. Scher C, Craig J, Nelson F (2008) Bone marrow edema in the knee in osteoarthrosis and association with total knee arthroplasty within a three-year follow-up. Skeletal Radiol 37(7):609-617

4. Caplan AI, Correa D (2011) The MSC: an injury drugstore. Cell Stem Cell 9(1):11-15

5. Caplan AI (2017) Mesenchymal stem cells: time to change the name! Stem Cells Transl Med 6(6):1445-1451

6. Eymard F, Parsons C, Edwards MH, Petit-Dop F, Reginster JY, Bruyère $O$, Richette $P$, Cooper $C$, Chevalier X (2015) Diabetes is a risk factor for knee osteoarthritis progression. Osteoarthritis Cartilage 23(6):851-859

Publisher's note Springer Nature remains neutral with regard to jurisdictional claims in published maps and institutional affiliations.

\section{Declarations}

Ethics approval APPENDIX A 